\title{
Outils numériques de maîtrise des risques vis-à-vis de l'environnement
}

\author{
par Farrokh Fotoohi, \\ Lyonnaise des Eaux, CIRSEE \\ Thierry Denoeux, \\ UTC Compiègne
}

Software tools for mastering environmental risks

The impact of water quality on public health, the fragile and limited nature of the natural water resources and the stakes related to environmental management require the establishment of high performance tools for monitoring both the national resources and water treatment works. This paper presents the recent work carried out by Lyonnaise des Eaux in this domain and the importance of software tools for the analysis of data to understand and manage the risks relating to the environment.

\section{G GESTION DE L'EAU ET DE L'ENVIRONNEMENT}

La gestion de l'environnement et en particulier les ressources en eau constituent un enjeu important. L'impact de la qualité de l'eau sur la santé publique, la nature fragile et limitée des ressources en eau et les enjeux liés à la gestion de l'environnement ont conduit à l'établissement de réglementations de plus en plus rigoureuses concernant la qualité de l'eau potable distribuée et les rejets en milieu naturel.

Une gestion pérenne et globale de l'eau et de l'environnement doit prendre en compte l'ensemble des activités (domestiques, industrielles et agricoles) à l'échelle d'un bas$\sin$ versant. Les distributeurs d'eau potable et les gestionnaires des systèmes d'assainissement jouent un rôle important dans cette gestion globale. Ils sont responsables d'une part de la distribution d'une eau de bonne qualité et en quantité suffisante aux consommateurs et d'autre part de la collecte des eaux usées et de leur traitement.

La maîtrise des risques dans le domaine de l'eau est en premier lieu la maîtrise de la qualité sanitaire de l'eau potable et la prise en compte d'une exigence de disponibilité très élevée de la part des usagers, comparable à celle concernant la distribution d'électricité. C'est également la préservation à long terme des ressources en eau et la maîtrise des rejets en milieu naturel. C'est enfin l'intégration de tous ces aspects dans une démarche globale de maîtrise des coûts d'exploitation et d'investissement dans un contexte où la demande et les niveaux d'exigence ne cessent de croître.

Afin d'accompagner cette démarche globale, Lyonnaise des Eaux a développé depuis plusieurs années des outils numériques pour la maîtrise des risques dans son domaine d'activité et à différents niveaux : la surveillance du milieu naturel et des ouvrages, le pilotage des installations et la gestion à moyen et long termes des exploitations. Cette communication présente les travaux effectués essentiellement dans le cadre de la surveillance et gestion de l'environnement.

\section{II - SURVEILLANCE DE LA RESSOURCE ET DES OUVRAGES}

Afin de respecter leurs obligations, les distributeurs d'eau et les gestionnaires des systèmes d'assainissement sont amenés à effectuer une surveillance permanente de la qualité de la ressource (rivière, nappe souterraine...) et du fonctionnement des réseaux, des installations de traitement d'eau potable et des stations d'épuration.

Cette surveillance nécessite l'installation d'un grand nombre de capteurs géographiquement distribués générant un flux très important de données. Pour apprécier la situation et prendre une décision adéquate, l'exploitant a besoin d'informations pertinentes. Il doit donc disposer d'outils informatiques performants assurant non seulement la récupération des données et leur stockage, mais également leur validation et transformation en information fiable et synthétique.

Le projet ESPRIT EM2S (Environnemental Monitoring and Management System, 1996-1999) réalisé dans le cadre du programme européen Esprit, a permis de formaliser cette problématique, appelée en terme scientifique "fusion de données", et de développer un système de supervision intelligent. Le système intègre des méthodes innovantes de validation, de reconstruction et de classification de données. Grâce à une architecture logicielle modulaire, il permet de générer des applications adaptées aux différents domaines techniques du métier de l'eau. 
Les paramètres pris en compte dans le cadre de la surveillance de la ressource et du fonctionnement des installations sont en effet nombreux, en partie redondants (certains étant liés), peuvent éventuellement être manquants ou erronés en cas de défaillances de capteurs, de sorte que l'ensemble est difficilement directement exploitable par un opérateur. L'idée consiste d'une part à valider les données brutes en exploitant les corrélations afin de reconstruire les éventuelles données manquantes ou aberrantes, et d'autre part à élaborer une information synthétique permettant à l'exploitant de juger globalement de la situation.

\section{III $\square$ PRINCIPES DE VALIDATION ET DE FUSION DE DONNÉES}

La figure 1 illustre le principe d'un système de fusion de données tel qu'il a été développé dans le cadre du projet EM2S. L'objectif général d'un tel système est la transformation de données de bas niveau (brutes, par essence peu fiables, peu compréhensibles et redondantes) issues des capteurs, en une information de haut niveau c'est-à-dire validée, synthétique et directement exploitable par l'utilisateur. Cette transformation peut être décomposée en trois étapes :

- la validation des données ou la détection des valeurs erronées ou suspectes (dues par exemple à un dysfonctionnement des capteurs) :

- la reconstruction des valeurs manquantes en utilisant des relations statistiques entre les différents paramètres mesurés ; - l'élaboration d'une information de haut niveau au moyen de capteurs logiciels.

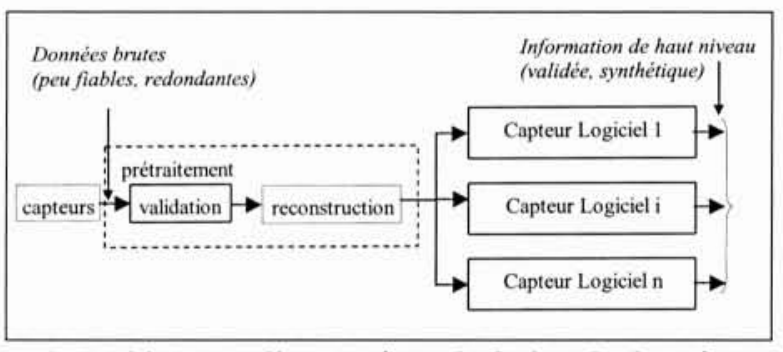

1. Architecture d'un système de fusion de données

\subsection{Validation des données}

La validation a pour but d'améliorer la robustesse du système de traitement des données vis-à-vis de dysfonctionnements de la chaîne d'acquisition des informations. Elle repose sur l'évaluation de la plausibilité de chaque observation d'un paramètre mesuré en tenant compte des observations effectuées dans le passé pour le même paramètre et/ou des corrélations connues avec d'autres paramètres. Il existe pour cela plusieurs approches : statistiques (estimation de densité), floues (combinaison de degrés de possibilité), et connexionnistes (réseaux de neurones).

La figure 2 illustre le principe de la validation monoparamètre d'un capteur $\mathrm{pH}$. Le signal et ses variations sont comparés à la gamme de valeurs typiquement obtenues en absence d'erreurs de mesure. Cette comparaison conduit au calcul d'un indice de confiance dans la validité de données à chaque instant. Les données dont l'indice de confiance est inférieur à un seuil sont considérées comme suspectes ou invalides.

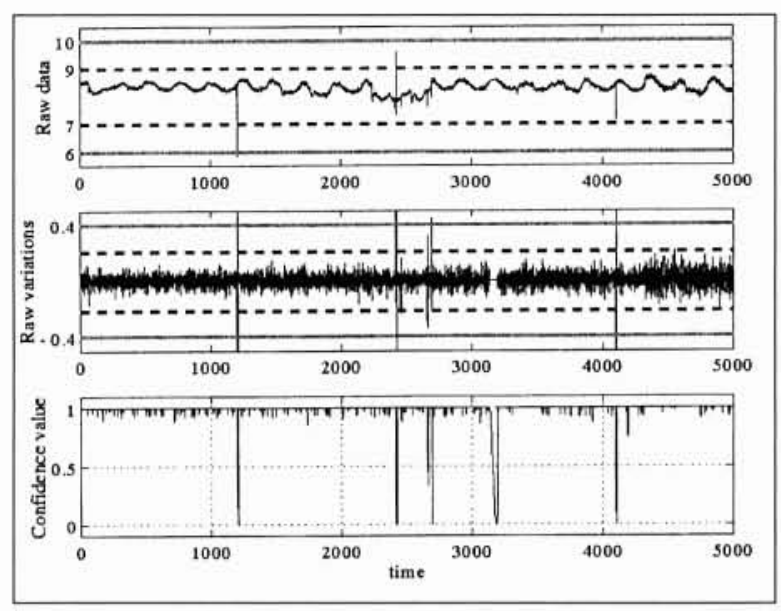

2. Validation monoparamètre d'un capteur de pH.

\section{- 3.2 Reconstruction des valeurs manquantes}

Cette étape consiste à exploiter l'information statistique contenue dans la base de données de manière à estimer les composantes manquantes d'un vecteur de mesures. Ce problème peut être traité dans un cadre probabiliste : il s'agit alors d'estimer les paramètres d'une loi de probabilité à partir de données comportant éventuellement elles-mêmes des valeurs manquantes, en utilisant par exemple l'algorithme EM (Expectation-Maximisation) ; les valeurs manquantes sont alors remplacées par leur loi de probabilité conditionnelle estimée, ou par un indicateur de position de cette loi.

Une autre approche $[1,2]$ consiste à s'appuyer sur un système neuro-flou construit par apprentissage à partir des données. Les valeurs manquantes sont alors remplacées par des nombres flous dont la fonction d'appartenance traduit l'incertitude associée à la reconstruction, et dont la propagation dans les calculs ultérieurs est plus facile que dans le cadre probabiliste. Dans les deux cas, la qualité de la reconstruction est évidemment fonction de la quantité d'information disponible ; elle doit impérativement être reflétée par la forme d'une distribution de valeur (de type probabiliste ou possibiliste), et non seulement par une valeur unique. La figure 3 illustre un exemple de données aberrantes générées artificiellement (dérive dans le temps d'un capteur d'oxygène dissous), ainsi que leur détection et leur reconstruction [3].

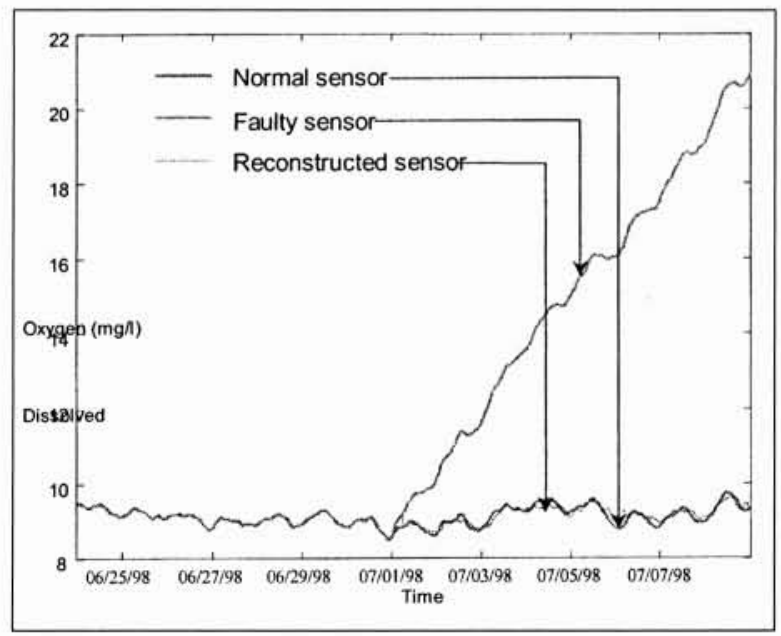

3. Exemple de données invalides détectées et reconstruites. 


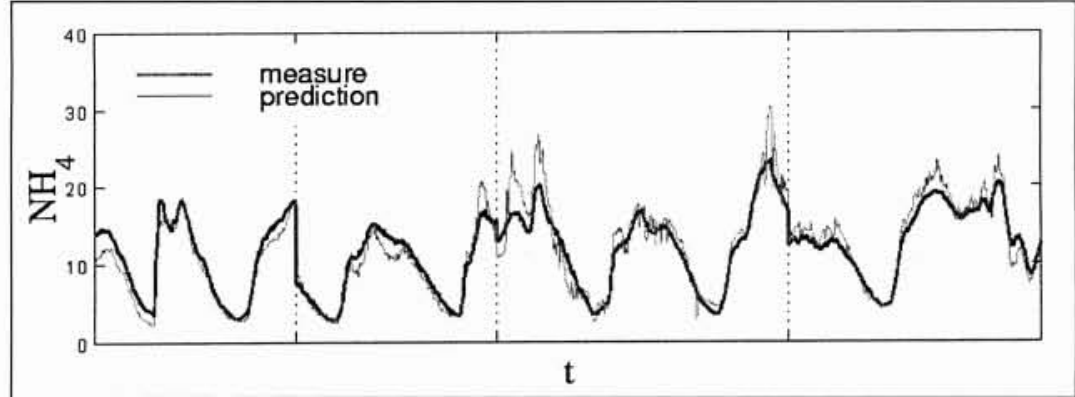

4. L'estimation de $\mathrm{NH}_{4}$ par réseau de neurone.

$\mathrm{pH}$, d'oxygène dissous, de température et de conductivité.

Un autre exemple est celui d'un réseau de neurones pour la prédiction du taux de coagulant dans une usine de production d'eau potable [4]. L'une des étapes les plus délicates dans le traitement des eaux de surface concerne en effet la clarification, qui consiste à éliminer les particules en suspension dans l'eau. La détermination de la dose de réactifs à injecter est délicate, le dosage optimal des réactifs étant lié de manière complexe aux caractéristiques de l'eau brute. Il est le plus sou-

\subsection{Capteurs logiciels}

Le processus d'élaboration, à partir de données validées et complétées, d'une information de haut niveau peut être structuré sous la forme de capteurs logiciels dont la fonction consiste à fournir à l'utilisateur une information complexe non observable directement, tel qu'un paramètre physicochimique non mesurable en continu (ou à un coût prohibitif), un indice global de qualité ou de traitabilité de l'eau, ou une consigne pour l'ajustement des procédés de traitement (taux de réactif). La construction d'un capteur logiciel peut être essentiellement vue comme un problème d'apprentissage de relations entre variables à partir de données observées, pour lequel les réseaux de neurones constituent un outil particulièrement performant. A titre d'exemple, la figure 4 montre la qualité de résultats obtenus par le réseau de neurones développé pour l'estimation de $\mathrm{NH}_{4}$ à partir des mesures de vent effectué manuellement : une fois par jour, on prélève un échantillon d'eau brute, et on détermine le dosage optimal en laboratoire (jar test), afin de déterminer une consigne de traitement jusqu'au lendemain.

Le réseau de neurones mis en place sur l'usine de Viry en Région Parisienne permet d'estimer automatiquement la dose de coagulant à injecter, à partir de mesures effectuées en continu sur l'eau brute. Une qualité fondamentale d'un tel système est sa robustesse par rapport à des erreurs de mesure ou à des modifications inattendues de la qualité de l'eau, d'où l'intérêt des méthodes de prétraitement (validation et reconstruction) mentionnées précédemment. La figure 5 montre en fonction du temps la dose de coagulant prescrite par le système avec et sans validation des variables d'entrée, démontrant ainsi la robustesse de la chaîne de traitement développée.

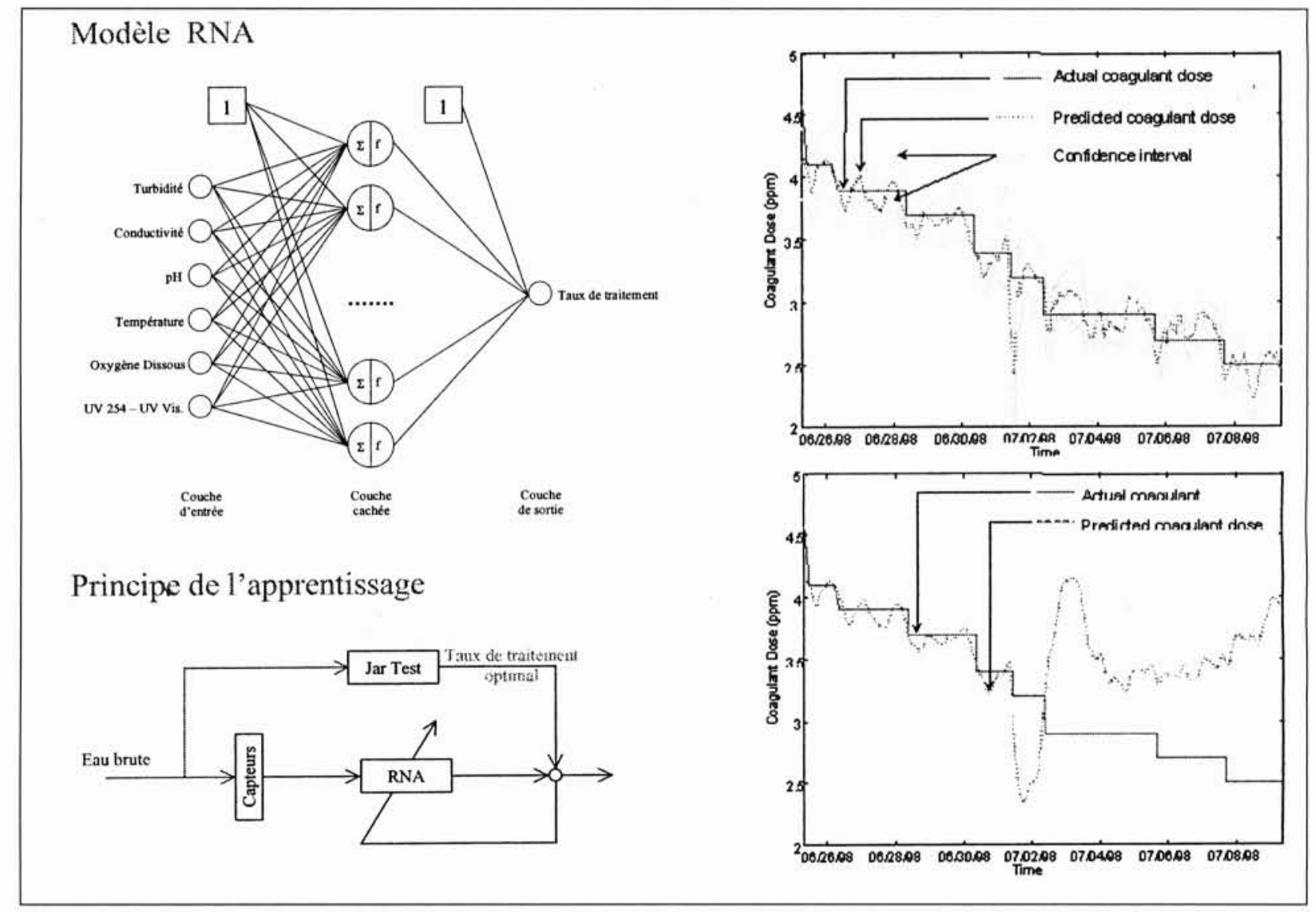

5. Dose de coagulant prescrite par le système (predicted coagulant dose) et par la méthode de référence : jar test (actual coagulant), avec (en haut) et sans (en bas) validation des données d'entrée. 


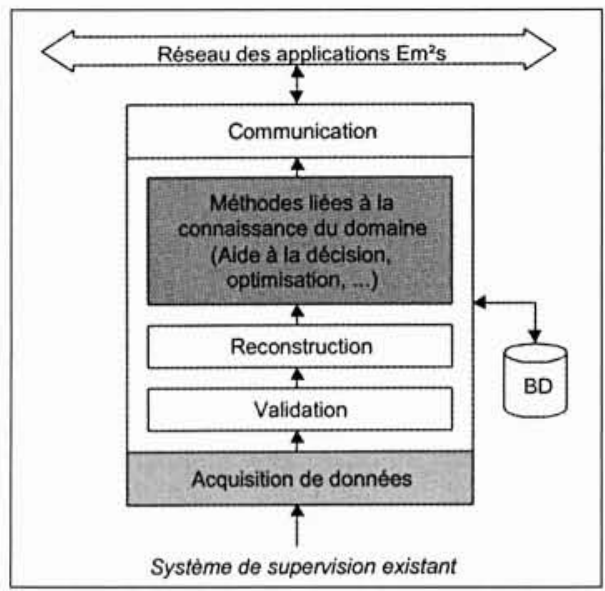

6. Architecture logiciel de la plate-forme EMS

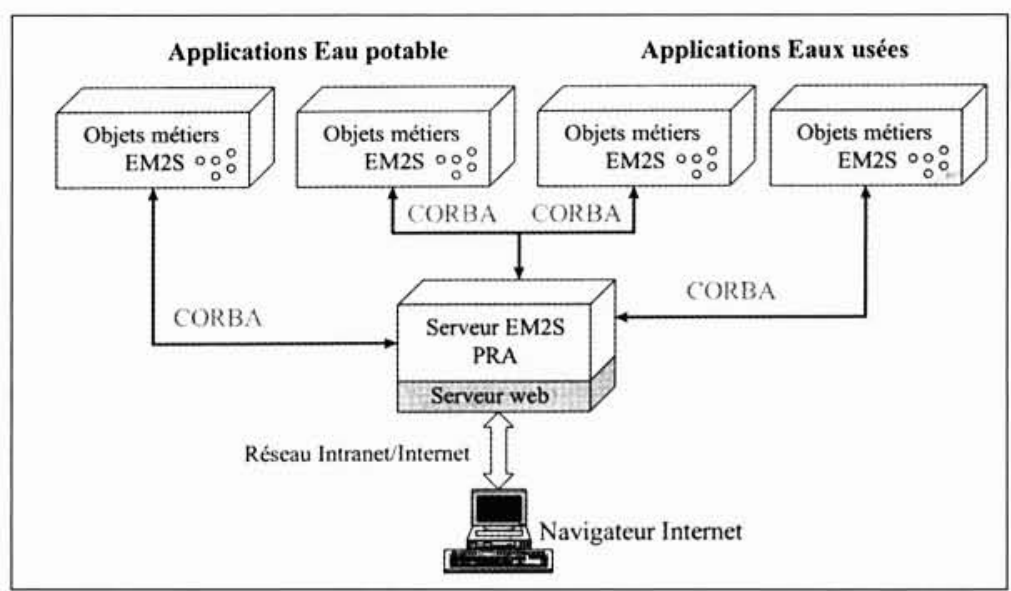

7. Principe de communication interapplications.

\section{IV - ARCHITECTURE INFORMATIQUE DU SYSTÈME}

La plate-forme $\mathrm{EM}^{2} \mathrm{~S}$ est un système informatique basé sur une architecture distribuée et modulaire. Elle permet de mettre en cuvre des applications pour la surveillance et la gestion du cycle de l'eau. La figure 6 illustre les différentes couches logicielles de cette plate-forme. Trois niveaux principaux sont à distinguer :

- Fonctions standards de surveillance : ces fonctions concernent la communication entre le système et la supervision et les bases de données externes, les modules standards de validation et de reconstruction de données, l'interface utilisateur ainsi que la base de données interne.

- Modules spécifiques par domaine d'application : il s'agit des méthodes de calculs qui sont définies pour chaque domaine. Par exemple, le calcul des volumes déversés par un déversoir d'orage est un calcul spécifique de l'application d'auto-surveillance des réseaux d'assainissement.

- Communication inter-applications : cette couche permet aux différentes applications $\mathrm{EM}^{2} \mathrm{~S}$ d'échanger des données et de partager des ressources à travers le réseau. La technologie utilisée est celle des objets distribués. Une partie de l'interface utilisateur du système étant écrite en JAVA, le système permet un accès en consultation à travers un navigateur Web (Intranet/Internet). Le schéma 7 illustre les principes de fonctionnement du système.

\section{V $\square$ APPLICATIONS}

A l'heure actuelle, la plate-forme EM'S est utilisée à la Lyonnaise des Eaux pour la mise en place de systèmes de surveillance intelligente dans différents domaines du cycle de l'eau : surveillance du milieu naturel, reporting qualité des usines de traitement, diagnostic du fonctionnement des stations d'épuration, ... Ces applications sont opérationnelles sur une dizaines de sites en France.

Le système a permis en particulier de mettre en œuvre le logiciel SANDRA (Système d'Autosurveillance Numérique des Réseaux d'Assainissement) dont l'objectif est la surveillance quotidienne des déversoirs du réseau de collecte et l'édition automatique des rapports réglementaires. Sandra permet de récupérer les données provenant des différents capteurs présents sur le réseau de collecte : pluviomètres, hauteurs, vitesses, débits, ... Les modules de validation automatique des

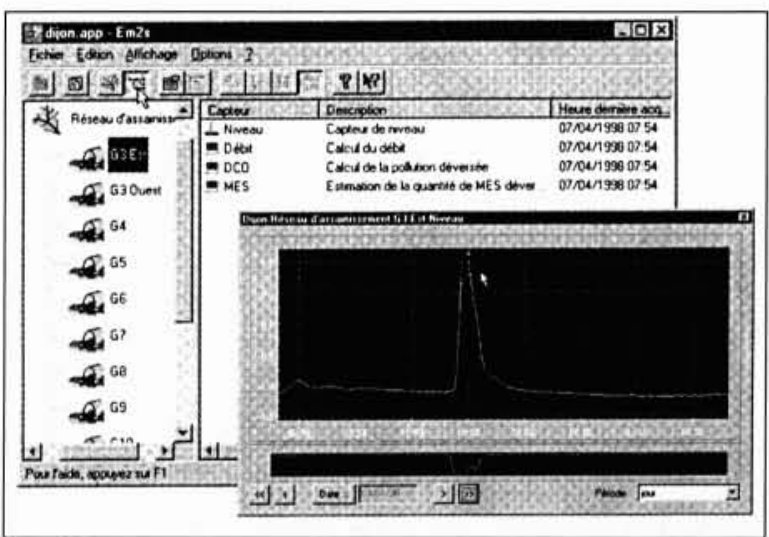

8. Ecrans de SANDRA (application EM'S pour l'autosurveillance des réseaux d'assainissement).

données apportent une aide appréciable aux exploitants pour la détection des anomalies de fonctionnement de la chaîne de mesure. A partir des données validées, Sandra permet de générer différents bilans de fonctionnement. En particulier, elle permet l'édition du rapport réglementaire d'autosurveillance qui, pour chaque déversoir d'orage, calcule les volumes d'eau déversés et fait une estimation de la pollution (MES, DCO) rejetée dans le milieu naturel (figure 8).

Système opérationnel depuis 1997 à la ville de Dijon, il est devenu l'outil standard de Lyonnaise des Eaux et est intégré dans l'offre commerciale Lyonnaise des Eaux aux collectivités locales.

\section{RÉFÉRENCES}

[1] S. Petit-Renaud and T. Denoeux A : neuro-fuzzy system for missing data reconstruction. IEEE Workshop on Emerging Technologies, Intelligent Measurement and Virtual systems for instrumentation and measurement, Saint-Paul USA, May 1998.

[2] S. Petit-Renaud and T. Denoeux A Fuzzy-neuro system for reconstruction of multi-sensor information, Fuzzy-Neuro Systems'98, Ed. W. Brauer Munich March 1998, Infix pages 322-329.

[3] T. J. Bohme, N. Valentin, C. S. Cox and T. Denoeux. : Comparison of autoassociative neural networks and Kohonen maps for signal failure detection and reconstruction. Proceedings of ANNIE'99 Saint-Louis. November 1999.

[4] N.VAlentin, F. Fotoohi and T. Denoeux : Modeling of coagulant dosing in a water treatment plant. EANN'99 Varsovie, September 1999. 